\title{
CONFORMAL MAPPINGS FOR CERTAIN DOUBLY CONNECTED DOMAINS*
}

\section{Br J. A. McFADDEN (University of Michigan)**}

1. Introduction. In this paper conformal mappings are derived for some doubly connected domains, the inner boundary being formed by slots and the outer boundary being the unit circle. All these mapping functions utilize elliptic functions; those for the more complicated domains involve elliptic functions of two different moduli. The domains appear, for example, in the linearized theory of supersonic flow past conical bodies.

2. Mapping of domains bounded by a slot and the unit circle. Consider first an annular domain in the $\zeta_{1}$-plane, $r_{1}<\left|\zeta_{1}\right|<1$, and the corresponding rectangular domain in the $z_{1}$-plane, $-2 K_{1}<x_{1}<2 K_{1}, 0<y_{1}<K_{1}^{\prime}$, obtained by a logarithmic transformation from the $\zeta_{1}$-plane. (See Fig. 1. Circular domains will be designated by Greek letters, $\zeta_{i}=\xi_{i}+i \eta_{i}$, and rectangular domains by Roman letters, $z_{i}=x_{i}+i y_{j}$.)

$$
z_{1}=\left(2 K_{1} / \pi i\right) \log \zeta_{1} \quad\left(0<\arg \zeta_{1}<2 \pi\right)
$$

The radius $r_{1}$ of the inner circle in the $\zeta_{1}$-plane is given by the expression

$$
r_{1}=\exp \left(-\pi K_{1}^{\prime} / 2 K_{1}\right) \text {. }
$$

The circle $\left|\zeta_{1}\right|=r_{1}$ can be collapsed onto a horizontal slot, $-\left(1-k_{1}^{\prime}\right) / k_{1} \leq \xi^{\prime} \leq$ $\left(1-k_{1}^{\prime}\right) / k_{1}, \eta^{\prime}=0$, (see Fig. 1), with the circle $\left|\zeta_{1}\right|=1$ mapping onto the circle $\left|\zeta^{\prime}\right|=1$, by means of the transformation

$$
\zeta^{\prime}=\frac{\operatorname{cn}\left(z_{1} ; k_{1}\right)+i k_{1}^{\prime} \operatorname{sn}\left(z_{1} ; k_{1}\right)}{\operatorname{dn}\left(z_{1} ; k_{1}\right)},
$$

where sn $\left(z_{1} ; k_{1}\right)$, cn $\left(z_{1} ; k_{1}\right)$, and $\mathrm{dn}\left(z_{1} ; k_{1}\right)$ are the Jacobian elliptic functions with argument $z_{1}$ and modulus $k_{1}$, and where $k_{1}^{\prime}=\left(1-k_{1}^{2}\right)^{1 / 2}$. (See, for example, reference [1].) The constants $K_{1}$ and $K_{1}^{\prime}$ in equation (2) may then be identified as the complete elliptic integrals of the first kind having moduli $k_{1}$ and $k_{1}^{\prime}$, respectively. An alternative form of equation (3) is the following:

$$
\begin{gathered}
-\left(1+\zeta^{\prime 2}\right) / 2 \zeta^{\prime}=\operatorname{sn}\left(z_{1}-K_{1} ; k_{1}\right) \\
\left(-2 K_{1}<x_{1}<2 K_{1} ; \quad 0<y_{1}<K_{1}^{\prime}\right)
\end{gathered}
$$

In this form we can understand the reason for the appearance of the Jacobian elliptic functions. The left member of (3a) maps the two boundaries in the $\zeta^{\prime}$-plane onto the real axis of an intermediate plane. The right member of $(3 \mathrm{a})$ maps the rectangular boundary in the $z_{1}$-plane onto the same real axis, since the sn-function is real on all four sides of the rectangle.

Note: This transformation is related to the transformation $\zeta^{\prime}=-\left(k_{0}\right)^{1 / 2}$ sn $\left(z_{0} ; k_{0}\right)$ by a Gauss transformation of elliptic functions (see, for example, reference [2]) and a translation of the coordinate axes. In the aerodynamic application mentioned above, the form (3) is found to be much simpler. The related form is discussed by Holzmüller [3].

*Received April 6, 1951. This paper is a chapter of a dissertation submitted in partial fulfillment of the requirements for the degree of Doctor of Philosophy at the University of Michigan, December, 1950. The author is grateful to Dr. R. C. F. Bartels for his many helpful suggestions.

**Now at U. S. Naval Ordnance Laboratory, White Oak, Maryland. 
The transformation (3) and equivalent combinations of functions have been used by previous investigators in the treatment of the linearized supersonic flow past a delta wing. (See, for example, reference [4].) We shall use it here as a basic transformation, from which more complicated transformations can be derived.
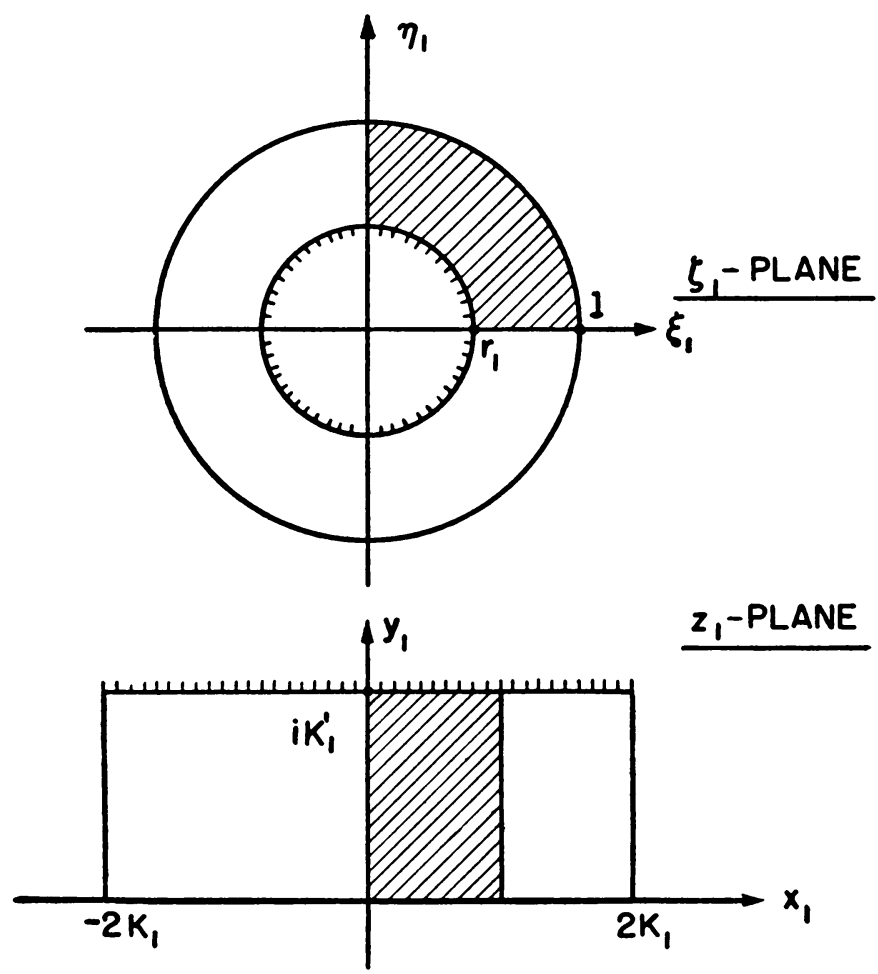

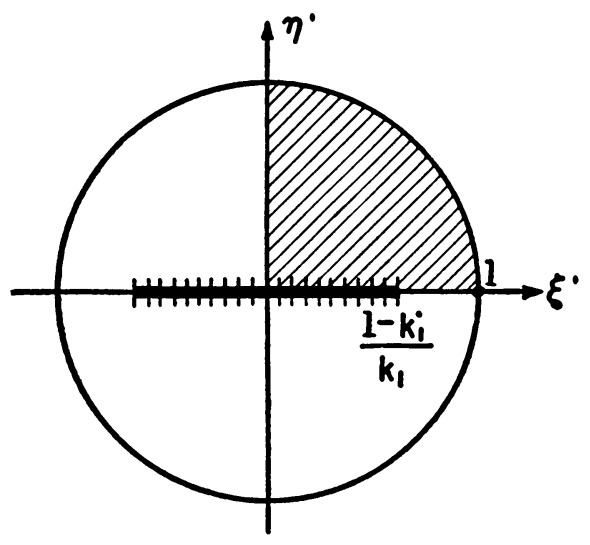

ל'-PLANE

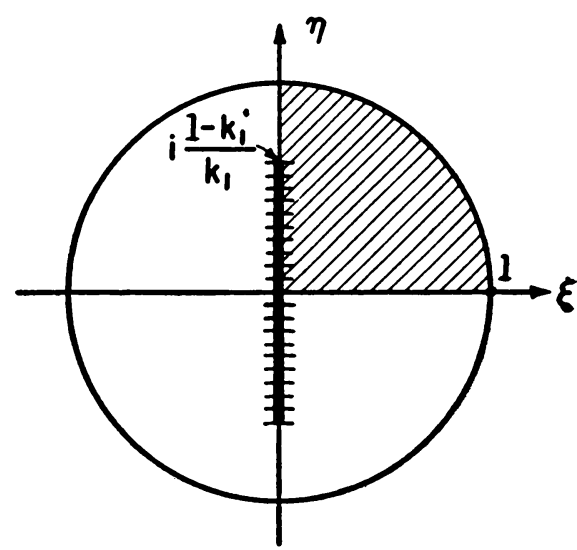

5-PLANE

FIG. 1. Conformal Mapping of Domains Bounded by a Slot and the Unit Circle. 
In equation (3) we may replace $\zeta^{\prime}$ by $i \zeta$ and $z_{1}$ by $\left(z_{1}+K_{1}\right)$. The resulting transformation collapses the circle $\left|\zeta_{1}\right|=r_{1}$ onto a vertical slot $\xi=0,-\left(1-k_{1}^{\prime}\right) / k_{1} \leq \eta \leq$ $\left(1-k_{1}^{\prime}\right) / k_{1}$ (see Fig. 1). The transformation function is

$$
\zeta=\mathrm{cn}\left(z_{1} ; k_{1}\right)+i \operatorname{sn}\left(z_{1} ; k_{1}\right) .
$$

An alternative form of equation (4) is the following:

$$
\begin{gathered}
i\left(1-\zeta^{2}\right) / 2 \zeta=\operatorname{sn}\left(z_{1} ; k_{1}\right) \\
\left(-2 K_{1}<x_{1}<2 K_{1} ; \quad 0<y_{1}<K_{1}^{\prime}\right)
\end{gathered}
$$

The transformation (1), followed by either (3) or (4), leaves the included segments of both axes invariant as well as the unit circle. (See Fig. 1.)

3. Mapping of domains bounded by a cross and the unit circle. Because of the invariance of the axes, the horizontal slot may be opened into a circle with symmetric horizontal fins in the $\zeta_{3}$-plane (see Fig. 2). We apply the inverse of transformations (1) and (2), but with a smaller modulus $k_{3}<k_{1}$. That is, let

$$
\zeta^{\prime}=\frac{\mathrm{cn}\left(z_{3} ; k_{3}\right)+i k_{3}^{\prime} \operatorname{sn}\left(z_{3} ; k_{3}\right)}{\operatorname{dn}\left(z_{3} ; k_{3}\right)},
$$

where

$$
z_{3}=\left(2 K_{3} / \pi i\right) \log \zeta_{3}, \quad\left(0<\arg \zeta_{3}<2 \pi\right) .
$$

The corresponding domain in the $z_{3}$-plane is a slotted rectangle (see Fig. 2).

The radius of the inner circle in the $\zeta_{3}$-plane is $r_{3}$, given by the equation

$$
r_{3}=\exp \left(-\pi K_{3}^{\prime} / 2 K_{3}\right) .
$$

An alternative form of equation (5) can be written, similar to equation (3a).

$$
\begin{gathered}
-\left(1+\zeta^{\prime 2}\right) / 2 \zeta^{\prime}=\operatorname{sn}\left(z_{3}-K_{3} ; k_{3}\right) \\
\left(-2 K_{3}<x_{3}<2 K_{3} ; \quad 0<y_{3}<K_{3}^{\prime}\right)
\end{gathered}
$$

If we combine equations (3a) and (5a), we may map directly from the $z_{1}$-plane to the $z_{3}$-plane. The resulting transformation may be written in four forms: (Related functions have been given by Kronsbein [5].)

$$
\begin{gathered}
\operatorname{sn}\left(z_{1}-K_{1} ; k_{1}\right)=\operatorname{sn}\left(z_{3}-K_{3} ; k_{3}\right) \\
\operatorname{cn}\left(z_{1}-K_{1} ; k_{1}\right)=\operatorname{cn}\left(z_{3}-K_{3} ; k_{3}\right) \\
\operatorname{dn}\left(z_{1}-K_{1} ; k_{1}\right)= \pm\left[1-k_{1}^{2} \operatorname{sn}^{2}\left(z_{3}-K_{3} ; k_{3}\right)\right]^{1 / 2} \\
\pm\left[1-k_{3}^{2} \operatorname{sn}^{2}\left(z_{1}-K_{1} ; k_{1}\right)\right]^{1 / 2}=\operatorname{dn}\left(z_{3}-K_{3} ; k_{3}\right) \\
\left(-2 K_{1}<x_{1}<2 K_{1} ; 0<y_{1}<K_{1}^{\prime} ;-2 K_{3}<x_{3}<2 K_{3} ; 0<y_{3}<K_{3}^{\prime}\right)
\end{gathered}
$$

If we argue in similar manner with equation (4a), we may construct a domain bounded internally by a circle with symmetric vertical fins in the $\zeta_{2}$-plane. (See Fig. 2.) 




FIG. 2. Conformal Mapping of Domains Bounded by a Cross and the Unit Circle. 
Again a new modulus $k_{2}<k_{1}$ is used. The resulting transformation may be written in four forms:

$$
\begin{gathered}
\operatorname{sn}\left(z_{1} ; k_{1}\right)=\operatorname{sn}\left(z_{2} ; k_{2}\right) \\
\operatorname{cn}\left(z_{1} ; k_{1}\right)=\operatorname{cn}\left(z_{2} ; k_{2}\right) \\
\operatorname{dn}\left(z_{1} ; k_{1}\right)= \pm\left[1-k_{1}^{2} \operatorname{sn}^{2}\left(z_{2} ; k_{2}\right)\right]^{1 / 2} \\
\pm\left[1-k_{2}^{2} \operatorname{sn}^{2}\left(z_{1} ; k_{1}\right)\right]^{1 / 2}=\operatorname{dn}\left(z_{2} ; k_{2}\right) \\
\left(-2 K_{1}<x_{1}<2 K_{1} ; 0<y_{1}<K_{1}^{\prime} ;-2 K_{2}<x_{2}<2 K_{2} ; 0<y_{2}<K_{2}^{\prime}\right)
\end{gathered}
$$

where

$$
z_{2}=\left(2 K_{2} / \pi i\right) \log \zeta_{2}, \quad\left(0<\arg \zeta_{2}<2 \pi\right) .
$$

The corresponding domain in the $z_{2}$-plane is again a slotted rectangle. (See Fig. 2.) The radius of the inner circle in the $\zeta_{2}$-plane is $r_{2}$, given by the equation

$$
r_{2}=\exp \left(-\pi K_{2}^{\prime} / 2 K_{2}\right) \text {. }
$$

A transformation similar to equation (3) will collapse the circle $\left|\zeta_{2}\right|=r_{2}$ onto the real axis in the $\zeta_{4}$-plane, leaving the vertical fins on the imaginary axis. (See Fig. 2.) The transformation equation is the following:

$$
\zeta_{4}=\frac{\operatorname{cn}\left(z_{2} ; k_{2}\right)+i k_{2}^{\prime} \operatorname{sn}\left(z_{2} ; k_{2}\right)}{\operatorname{dn}\left(z_{2} ; k_{2}\right)}
$$

A transformation similar to equation (4) will collapse the circle $\left|\zeta_{3}\right|=r_{3}$ onto the imaginary axis in the $\zeta_{4}$-plane, leaving the horizontal fins on the real axis. (See Fig. 2.) The transformation equation is the following:

$$
\zeta_{4}=\operatorname{cn}\left(z_{3} ; k_{3}\right)+i \text { sn }\left(z_{3} ; k_{3}\right)
$$

The two definitions of $\zeta_{4}$ in equations (12) and (13) are equivalent if and only if the moduli are related by the equation

$$
k_{1}^{\prime}=k_{2}^{\prime} k_{3}^{\prime} .
$$

[This statement can easily be proved in the form (17).] Then the inner boundary in the $\zeta_{4}$-plane is a cross consisting of the horizontal slot, $-\left(1-k_{2}^{\prime}\right) / k_{2} \leq \xi_{4} \leq\left(1-k_{2}^{\prime}\right) / k_{2}$, $\eta_{4}=0$, and the vertical slot, $\xi_{4}=0,-\left(1-k_{3}^{\prime}\right) / k_{3} \leq \eta_{4} \leq\left(1-k_{3}^{\prime}\right) / k_{3}$.

By a series of steps we have mapped the circle $\left|\zeta_{1}\right|=r_{1}$ (Fig. 1) onto a cross, the unit circle remaining invariant (as well as the coordinate axes).

It is convenient to introduce a shape factor $\epsilon$ for the cross, defined by the relation

$$
\epsilon=k_{2}^{\prime} /\left(k_{1}^{\prime}\right)^{1 / 2}=\left(k_{1}^{\prime}\right)^{1 / 2} / k_{3}^{\prime} .
$$

$\epsilon$ has the range $\left(k_{1}^{\prime}\right)^{1 / 2} \leq \epsilon \leq\left(k_{1}^{\prime}\right)^{-1 / 2}$. Then the moduli $k_{2}$ and $k_{3}$ and the respective complementary moduli are given in terms of $\epsilon$ and $k_{1}$ by the equations

$$
\begin{array}{ll}
k_{2}=\left(1-\epsilon^{2} k_{1}^{\prime}\right)^{1 / 2}, & k_{2}^{\prime}=\epsilon\left(k_{1}^{\prime}\right)^{1 / 2}, \\
k_{3}=\left(1-k_{1}^{\prime} / \epsilon^{2}\right)^{1 / 2}, & k_{3}^{\prime}=\left(k_{1}^{\prime}\right)^{1 / 2} / \epsilon .
\end{array}
$$


If $\epsilon=1$, then $k_{2}^{\prime}=k_{3}^{\prime}=\left(k_{1}^{\prime}\right)^{1 / 2}$ and the vertical fins have the same length as the horizontal fins. If $\epsilon=\left(k_{1}^{\prime}\right)^{-1 / 2}$, then $k_{2}=0, k_{3}=k_{1}, r_{2}=0$, and equations (8) become an identity. Then the cross degenerates to a vertical slot. If $\epsilon=\left(k_{1}^{\prime}\right)^{1 / 2}$, then $k_{2}=k_{1}$, $k_{3}=0, r_{3}=0$, and equations (9) become an identity. Then the cross degenerates to a horizontal slot. In general, if $\epsilon>1$, the vertical fins are longer than the horizontal fins. If $\epsilon<1$, the horizontal fins are longer. (Fig. 2 is drawn for $\epsilon<1$.)

The combination of equations (9) and (12) or of equations (8) and (13) gives the resulting equation

$$
\zeta_{4}^{2}=\frac{\mathrm{cn}\left(z_{1} ; k_{1}\right)+i \epsilon\left(k_{1}^{\prime}\right)^{1 / 2} \operatorname{sn}\left(z_{1} ; k_{1}\right)}{\operatorname{cn}\left(z_{1} ; k_{1}\right)-i \epsilon\left(k_{1}^{\prime}\right)^{1 / 2} \operatorname{sn}\left(z_{1} ; k_{1}\right)}
$$

Although the function $\zeta_{4}^{2}$ is an elliptic function, the function $\zeta_{4}$ is not. It is doubly periodic, but it has branch points. In particular, there are branch points at the images of the point $\zeta_{4}=0$, the intersection of the cross. These points are defined by the equations

$$
z_{1}= \pm\left(\frac{1}{2} K_{1}+\tau\right)+i K_{1}^{\prime}, \quad \pm\left(\frac{3 K_{1}}{2}-\tau\right)+i K_{1}^{\prime} .
$$

[These points are the zeros of the functions dn $\left(z_{2} ; k_{2}\right)$ and $\mathrm{dn}\left(z_{3}-K_{3} ; k_{3}\right)$. See Fig. 2.] If $\epsilon \leq 1, \tau \geq 0$. If $\epsilon \geq 1, \tau \leq 0$. If $\epsilon=\left(k_{1}^{\prime}\right)^{1 / 2}$, then $\tau=\frac{1}{2} K_{1}$ and the branch points coalesce to form simple zeros at the points $z_{1}= \pm K_{1}+i K_{1}^{\prime}$, the zeros of the function (3). If $\epsilon=\left(k_{1}^{\prime}\right)^{-1 / 2}$, then $\tau=-\frac{1}{2} K_{1}$ and the branch points coalesce to form simple zeros at the points $z_{1}=i K_{1}^{\prime}, \pm 2 K_{1}+i K_{1}^{\prime}$, as in the function (4).

Let us derive the mapping from the $\zeta_{1}$-plane onto the $\zeta_{4}$-plane by a distinctly different method. First, we square the variable $\zeta_{1}$, making the two circles doubly covered, the inner one being of radius $r_{1}^{2}$. Let the appropriate modulus be $\bar{k}_{1}$, so that the corresponding complete elliptic integrals are related to the radius by the equation

$$
r_{1}^{2}=\exp \left(-\pi \bar{K}_{1}^{\prime} / 2 \bar{K}_{1}\right) .
$$

Second, apply the transformation (3) with modulus $\bar{k}_{1}$ in the two-sheeted plane, preserving the doubly covered unit circle and creating a quadruply covered horizontal slot. Third, extract the square root. The final boundaries are the simply covered unit circle and a cross with four fins of equal length. The resulting transformation may be written as follows: (A related function has been given by Kronsbein [5].)

$$
\left(\zeta_{4}^{\prime}\right)^{2}=\frac{\operatorname{cn}\left(\left[4 \bar{K}_{1} / \pi i\right] \log \zeta_{1} ; \bar{k}_{1}\right)+i \bar{k}_{1}^{\prime} \operatorname{sn}\left(\left[4 \bar{K}_{1} / \pi i\right] \log \zeta_{1} ; \bar{k}_{1}\right)}{\operatorname{dn}\left(\left[4 \bar{K}_{1} / \pi i\right] \log \zeta_{1} ; \bar{k}_{1}\right)}
$$

Comparison of equations (2) and (19) reveals that the moduli $k_{1}$ and $\bar{k}_{1}$ are related by a Landen transformation. (See, for example, reference [6].) If we apply the Landen transformation, whereby

$$
\begin{array}{ll}
\bar{k}_{1}=\left(1-k_{1}^{\prime}\right) /\left(1+k_{1}^{\prime}\right), & \bar{k}_{1}^{\prime}=2\left(k_{1}^{\prime}\right)^{1 / 2} /\left(1+k_{1}^{\prime}\right), \\
\bar{K}_{1}=\frac{1}{2}\left(1+k_{1}^{\prime}\right) K_{1}, & \bar{K}_{1}^{\prime}=\left(1+k_{1}^{\prime}\right) K_{1}^{\prime},
\end{array}
$$

and 


$$
\begin{aligned}
& \operatorname{sn}\left(\left[1+k_{1}^{\prime}\right] z_{1} ; \bar{k}_{1}\right)=\left(1+k_{1}^{\prime}\right) \frac{\operatorname{sn}\left(z_{1} ; k_{1}\right) \mathrm{cn}\left(z_{1} ; k_{1}\right)}{\operatorname{dn}\left(z_{1} ; k_{1}\right)} \\
& \operatorname{cn}\left(\left[1+k_{1}^{\prime}\right] z_{1} ; \bar{k}_{1}\right)=\frac{1-\left(1+k_{1}^{\prime}\right) \mathrm{sn}^{2}\left(z_{1} ; k_{1}\right)}{\operatorname{dn}\left(z_{1} ; k_{1}\right)} \\
& \operatorname{dn}\left(\left[1+k_{1}^{\prime}\right] z_{1} ; \bar{k}_{1}\right)=\frac{1-\left(1-k_{1}^{\prime}\right) \mathrm{sn}^{2}\left(z_{1} ; k_{1}\right)}{\operatorname{dn}\left(z_{1} ; k_{1}\right)}
\end{aligned}
$$

then equation (20) may be written

$$
\left(\zeta_{4}^{\prime}\right)^{2}=\frac{\operatorname{cn}\left(z_{1} ; k_{1}\right)+i\left(k_{1}^{\prime}\right)^{1 / 2} \operatorname{sn}\left(z_{1} ; k_{1}\right)}{\operatorname{cn}\left(z_{1} ; k_{1}\right)-i\left(k_{1}^{\prime}\right)^{1 / 2} \operatorname{sn}\left(z_{1} ; k_{1}\right)},
$$

where $z_{1}$ is given as in (1).

We observe that equation (23) is indeed the mapping function for a cross of equal lengths, for it is a special case of equation (17), namely $\epsilon=1$.

The cross of equal lengths can easily be mapped into the cross of unequal lengths in Fig. 2. We apply a linear fractional transformation which preserves the unit circle in the two-sheeted plane. If the transformation

$$
\zeta_{4}^{2}=\frac{\left(\zeta_{4}^{\prime}\right)^{2}-(\epsilon-1) /(\epsilon+1)}{1-[(\epsilon-1) /(\epsilon+1)]\left(\zeta_{4}^{\prime}\right)^{2}}
$$

is combined with equation (23), then equation (17) is the result.

\title{
REFERENCES
}

[1] E. T. Whittaker and G. N. Watson, Modern Analysis, Cambridge Univ. Press, London, 1927, ch. 22.

[2] R. Fricke, Die elliptische Funktionen, vol. 2, Leipzig, 1922, p. 293.

[3] G. Holzmüller, Einführung in die Theorie der isogonalen Verwandtschaften und der conformen Abbildung, Teubner, Leipzig, 1882, pp. 256-258.

[4] H. J. Stewart, The lift of a delta wing at supersonic speeds, Quart. Appl. Math. 4, 246-254 (1946).

[5] J. Kronsbein, Analytical expressions for some extremal schlicht functions, J. Lond. Math. Soc., 17, 152-157 (1942).

[6] Whittaker and Watson, loc. cit., pp. 507-508.

\section{EFFECT OF A RIGID ELLIPTIC DISK ON THE STRESS DISTRIBUTION IN AN ORTHOTROPIC PLATE*}

\author{
By A. J. OWENS AND C. B. SMITH, (University of Florida)
}

A thin orthotropic plate of uniform thickness will possess two perpendicular axes of symmetry in the plane of the plate. An infinite rectangular plate of this type containing a rigid elliptic disk with major and minor axes coinciding with the axes of symmetry is discussed. A uniform tension is assumed to act along two opposite edges of the plate and a mathematical analysis of the stress distribution is given. It is assumed the 